\title{
Dynamic behavior of Trigger Factor on the ribosome
}

Deeng, J. ${ }^{1}$, Chan, K.Y. ${ }^{2}$, van der Sluis, E.0. 1, Berninghausen, 0. 1, Han, W. ${ }^{2}$, Gumbart, J. ${ }^{3}$, Schulten, K. ${ }^{2}$, Beatrix, B. ${ }^{1, *}$ and R. Beckmann ${ }^{1, *}$

${ }^{1}$ Gene Center Munich and Center for integrated Protein Science Munich, Department of Biochemistry, Ludwig-Maximilians-Universität München, FeodorLynen-Straße 25, 81377 Munich, Germany

${ }^{2}$ Beckman Institute for Advanced Science and Technology, University of Illinois at Urbana-Champaign, 405 N. Mathews Ave, Urbana Illinois 61801, USA

${ }^{3}$ School of Physics, Georgia Institute of Technology, Atlanta, Georgia 30332, USA

*to whom correspondence should be addressed:

beckmann@genzentrum.Imu.de, beatrix@genzentrum.lmu.de 


\begin{abstract}
:
Trigger factor is the only ribosome-associated chaperone in bacteria. It interacts with hydrophobic segments in nascent chains as they emerge from the ribosome. TF binds via its N-terminal ribosome-binding domain (RBD) mainly to ribosomal protein uL23 at the tunnel exit on the large ribosomal subunit. Whereas earlier structural data suggested that $\mathrm{TF}$ binds as a rigid molecule to the ribosome, recent comparisons of structural data on substrate-bound, ribosome-bound and $\mathrm{TF}$ in solution from different species suggest that this chaperone is a rather flexible molecule. Here we present two cryo-EM structures of TF bound to ribosomes translating an mRNA coding for a known TF substrate from E. coli of different length. The structures reveal distinct degrees of flexibility for the different TF domains, a conformational rearrangement of the RBD upon ribosome binding and an increase in rigidity within TF when the nascent chain is extended. Molecular dynamics simulations agree with these data and offer a molecular basis for these observations.
\end{abstract}

\title{
Keywords:
}

Protein folding, chaperones, ribosome binding domain, cryo electron microscopy, molecular dynamics simulation.

\section{Abbreviations:}

$\mathrm{TF}$, trigger factor

$\mathrm{RBD}$, ribosome binding domain

SBD, Substrate binding domain

PPIase, peptidyl-prolyl cis/trans-isomerase

GatD, galactitol-1-phosphate dehydrogenase

$\mathrm{RAC}$, ribosome-associated complex

NAC, nascent polypeptide-associated complex

Ssb, heat shock protein SSB1

\section{Introduction:}

During translation the nascent polypeptide chain emerges from the ribosomal exit tunnel and is released into the crowded environment of the cytosol where 
processes such as protein folding and targeting for translocation must occur. These processes are assisted by protein factors such as the signal recognition particle (SRP) and its receptor (SR) in the case of targeting, and different chaperones and chaperonins in case of protein folding. Whereas in eukaryotes a whole repertoire of chaperones (such as RAC, NAC, Ssb) interacts with the ribosome near the tunnel exit on the large ribosomal subunit, in bacteria trigger factor (TF) is the only chaperone, that directly interacts with the ribosome. TF is composed of three domains: an N-terminal ribosome binding domain (RBD), the peptidyl-prolyl cis/trans-isomerase (PPIase) domain or head domain, and the Cterminal substrate binding domain (SBD) located between the RBD and PPIase domain in the TF structure and forming the arms 1 and 2 [1](see Supplementary figure 1). The RBD and the C-terminal domain harbor the main chaperone activity of TF, whereas the PPIase domain is dispensable for the chaperone activity in vivo [2, 3]. Nevertheless, more recent reports suggest that, at least for some proteins, the PPIase domain provides a second chaperone interaction site for the nascent chain [4-7]. Most of our knowledge about the involvement of chaperones in protein folding originates from in vitro studies, where the refolding of chemically denatured substrate proteins was monitored in the presence of chaperones with various techniques. However, in the recent years many studies have shown in eukaryotes as well as in prokaryotes that protein folding already begins on the ribosome, while the C-terminal part of the polypeptide is still in the process of being extended at the peptidyl-transferase center. Furthermore, several recent studies have shown that the formation of secondary structures, such as $\alpha$-helices, already begins within the ribosomal exit tunnel [8-15].

To date, three crystal structures of TF RBDs in complex with $50 \mathrm{~S}$ ribosomal subunits (one heterologous pair of E. coli TF and archaeal 50S from Haloarcula marismortui and two homologous pairs of $\mathrm{TF}$ and $50 \mathrm{~S}$ from Deinococcus radiodurans) have revealed the overall positioning of the RBD on empty ribosomes $[1,16,17]$. Docking of the crystal structure of a complete TF molecule onto the structure of the 50S associated RBD indicated that the chaperone is localized in a position so that it "arches" over the ribosomal tunnel exit [1]. A cryo-EM structure of E. coli ribosomes carrying a stalled nascent chain of a non- 
physiological substrate (SH3, which can fold independently of TF) with a TF molecule fixed to the nascent chain via a covalent disulfide bond in close proximity to the tunnel exit, confirmed the localization of the chaperone over the ribosomal tunnel exit [18]. Nevertheless, due to the limited resolution of this structure (19 $)$, the interaction of TF with the nascent chain was not studied in molecular detail. Therefore, we aimed to use cryo-EM to visualize the ribosomebound TF when interacting with a physiological substrate emerging from the ribosomal tunnel. For this purpose we choose the galactitol-1-phosphate dehydrogenase (GatD) as the nascent polypeptide chain because it is known to interact with TF on the ribosome and exhibits two hydrophobic stretches $[6,19]$. Here we show, that TF interacts with ribosome nascent chain complexes (RNCs) carrying this substrate with high affinity. The cryo-EM structure of the TFGatD85-RNC exhibits a sub-nanometer resolution for the ribosome and the Nterminal RBD of TF, whereas the middle domain and the head (PPIase) domain of TF are less-well resolved due to increasing flexibility along the molecule. The average resolution of our TF-GatD85-RNC map (7.7 ̊) enabled us to visualize the nascent chain within the ribosomal tunnel and to even trace it onto the surface of the TF-RBD. We observe the two alpha helices of the RBD to undergo a conformational change when bound to the translating ribosome. This rearrangement causes the presentation of a new hydrophobic surface on the RBD, which serves as interaction site for hydrophobic stretches within the emerging nascent chains. Using microscale thermophoresis we demonstrate that the affinity of TF for RNCs increases as the nascent chain is elongated that may reflect an altered conformation of TF. We confirm this finding with a second TFRNC cryo-EM structure, where TF in complex with RNCs carrying a longer nascent chain of GatD (145 amino acids total length) gained rigidity partially due to additional interactions of TF with either the nascent chain or the ribosomal surface. Molecular dynamics simulations reveal the molecular interactions between TF and the nascent chain, elucidating how the length of the nascent chain modulates the conformation of RNC-bound TF and the binding/release of TF with RNCs.

\section{Results:}


We generated RNCs carrying an 85 amino acid (total length) long GatD nascent polypeptide stalled by the TnaC stalling sequence in vivo (Fig. $1 \mathrm{a}$ and supplementary table 1) [20,21]. In order to enable purification and detection of the RNCs, the used mRNA coded for an N-terminal His-tag followed by an HA tag followed by F61 to C103 of GatD and the stalling sequence of TnaC. The purified GatD85-RNCs showed a homogenous population of ribosomes carrying only peptidyl-tRNAs. Free peptide of the nascent chain was not visible, indicating stably stalled RNCs [22]. Binding of TF to these GatD85-RNCs was shown using microscale thermophoresis (see below). We reconstituted purified GatD85-RNCs with TF and subjected these complexes to cryo-EM and single particle analysis following standard procedures[23]. After several rounds of global and focused sorting of the dataset a stable population of 100,931 particles was refined to an average resolution of $7.7 \AA ̊$ (FSC 0.5 criterion, see supplemental Figure 2a). The resulting structure resembled a programmed $70 \mathrm{~S}$ ribosome with additional density for the P-site tRNA as expected, as well as the nascent chain and density representing $\mathrm{TF}$ at the ribosomal tunnel exit (Fig. $1 \mathrm{~b}-\mathrm{d}$ ). This density did not account for the entire TF molecule but rather represented only parts of the RBD of TF. Nevertheless, an analysis of the local resolution revealed that the RBD showed a resolution distribution comparable to that of the ribosome itself, indicating a near stoichiometric occupation of the GatD85-RNCs with TF (Supplementary Fig. 2b). When we low pass filtered the same density map to a resolution of $16.5 \AA$ the C-terminal domain with its arms became visible (red mesh in Fig. 1), whereas the PPIase domain remained invisible, indicating a gradual increase in flexibility within TF from the RBD over the C-terminal domain towards the PPIase domain. The crystal structure of full length TF exhibits two possible pivot points that are likely to explain the observed flexibility. The RBD is connected via an extended unstructured loop (V111 to T133) with the PPIase domain. This loop is packed against another loop connecting the long $\alpha$-helix forming the back with arm1 (D299 to P303). The second pivot point may be located in the unstructured loops (A149 to K154 and E241 to L248) connecting the PPIase domain with the remaining of the TF molecule (Supplementary Fig. 1 and 5 a). These pivot points are in agreement 
with a normal mode analysis of the TF crystal structure (PDB ID: 1W26) and also with our MD simulations (see below).

Despite the high degree of flexibility of the C-terminal and the PPIase domain, the sub-nanometer resolution for the RBD and the ribosome enabled us to isolate the density for the nascent chain by comparing our density map with an empty 70S ribosome [21]. We detected density for the nascent chain along almost the entire length of the ribosomal tunnel (Fig. $1 \mathrm{~d}$ ). The path of the TnaC part of nascent chain here is consistent with a previous reconstruction [21]. In addition, we observed a compaction of the nascent chain $\mathrm{N}$-terminally of the stalling peptide. This compaction was localized below the constriction formed by the ribosomal proteins uL4 and uL22, a region of the tunnel which allows $\alpha$-helix formation [8-11]. This feature coincides with $\alpha$-helical conformation in a recently published GatD crystal structure from E. coli (PDB: 4UEJ residues C92 to C103)[24]. We therefore concluded that the compaction is the result of $\alpha$-helix formation within this region of the tunnel (see Fig. 2 a). Since this $\alpha$-helix is encoded directly C-terminal to the hydrophobic stretch1 in GatD (AA81 to 91 in original GatD amino acid sequence), it is likely that the lower part of the nascent chain density corresponds to this hydrophobic stretch (Fig. 2a).

For a molecular interpretation of the extra density at the tunnel exit we attempted to rigidly fit the crystal structure of E. coli TF RBD in solution (PDB ID: 10MS, chain A) [25] into the density. The RBD structure was oriented with respect to the ribosome according to the crystal structure of TF RBD in complex with the 50S ribosomal subunit from Deinoccocus radiodurans (PDB: 2D30)[17]. Even though the two characteristic $\alpha$-helices (1 and 2) of the TF RBD were clearly resolved in our density map, the crystal structure did not fit well. In solution, helix 2 shows a kink (between T58 and R63)[25], however upon binding to the ribosome, this kink is absent resulting in a prolonged helix 2 . In addition, helix 2 is shifted relative to helix 1. To explain our density, helix 1 and helix 2 were adjusted by shifts into the electron density using the Coot software [26] to fit to the density (see Fig. $2 \mathrm{~b}$ and supplementary figure 3). Since the loop connecting helix 1 and 2 was not unambiguously resolved, it was modeled according to (i) the EM density, (ii) the loop in structure (PDB ID: 2D30) and (iii) the known interactions between TF and the ribosome (TF_Arg45 interacts with 
uL23_Glu18 and 23S_A1392, TF_Arg57 with uL29_Ala33 and TF_Gln56 with uL29_Gln36) $[1,16,17]$ and the whole model was again regularized with respect to back bone geometry using Coot.

In our cryo-EM reconstruction, the rearranged helices are localized directly where the nascent chain first encounters the RBD of TF (see Fig. 2 c). When we analyzed the distribution of hydrophobic surface on the rearranged RBD in comparison to that of the TF RBD in solution (PDB ID: 10MS), we realized that the rearrangement of the two helices caused a presentation of a much larger hydrophobic surface (see Fig. 2 d). This is where TF first interacts with the hydrophobic stretch of GatD. The possibility that such a rearrangement might lead to the formation of a hydrophobic "landing surface" for the nascent chain was already suggested by Schluenzen et al. and our structure now supports the notion that this may indeed happen in the presence of a substrate nascent chain. An early encounter between the emerging nascent chain and TF via the landing surface of the RBD is also in agreement with chemical cross-linking data [18]. In order to determine whether the high degree of flexibility in our TF structure was due to the short length of the GatD nascent chain (85 amino acids including His- and HA-tag for purification and detection, and TnaC peptide for stalling) we prepared another sample of GatD-RNCs, where we extended the GatD nascent chain to a total length of 145 amino acids (GatD145-RNC) by adding the complete N-terminal sequence information of GatD while keeping the sequence context in and at the ribosomal tunnel exactly the same (see Fig. 3 a and supplementary table 1 ).

We used microscale thermophoresis, a technique which has been shown to provide comparable results for protein-protein interactions as surface plasmon resonance or fluorescence anisotropy [27], to determine whether the affinity of TF for GatD145-RNCs differed from that for GatD85-RNCs. For this purpose, we purified a TF variant carrying a unique cysteine at position 150 (located between the C-terminal and the PPIase domain, see supplementary fig 1). It was previously shown that mutation and labelling at this position does not change TF activity[5]. We labeled the cysteine with Alexa568. Upon titration of unlabeled RNCs, thermophoresis analysis revealed that the affinity of TF for binding to GatD145-RNCs (22 \pm 3 nM) was fivefold higher than for binding to GatD85-RNCs 


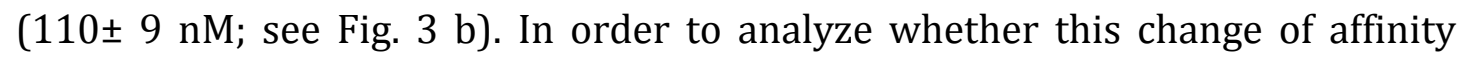
correlates with an altered conformation of TF on the ribosome, we determined a second cryo-EM reconstruction of a TF-GatD145-RNC complex.

Earlier crosslinking studies had already shown that nascent chains of >90aa length can be crosslinked to the PPIase (head) domain of TF [18]. We speculated that the GatD145-RNC nascent chain would be long enough to interact with the PPIase domain and thereby might reduce the flexibility of TF since it would provide more possible interaction sites between TF and the nascent chain. After several rounds of global and focused sorting of the dataset a stable population of 145,881 particles was refined to an overall average resolution of 7.8 A (FSC 0.5 criterion), a comparable resolution to that of the TF- GatD85-RNC structure (7.7 $\AA$, FSC 0.5 criterion). The presence of the GatD145 nascent polypeptide was again confirmed by additional density extending from the P-site tRNA into the ribosomal tunnel exit. In comparison to the TF-GatD85-RNC, more density for TF was visible in the TF-GatD145-RNC structure. Low pass filtered maps (10.5 $)$ displayed sufficient density to accommodate the full length TF indicating indeed a less flexible state of the head domain in this complex.

While the resolution of the head domain was too low to resolve secondary structure elements, its general location is in overall agreement with the previously published structure of TF covalently linked via a disulfide bridge to RNCs containing a folded SH3 domain [18]. Further sub-sorting, however, resulted in two sub-populations that differed with respect to the conformation of the head domain (see Fig. $3 \mathrm{c}$ and supplementary figure 4). While both TF conformations placed the PPIase domain in close proximity to the ribosomal surface, the exact positions where transient contacts to the ribosomal surface might be formed differed. Namely, in conformation 1 (35, 223 particles, average resolution $9.1 \AA$ ), one arm of the TF could make a contact to helix 24 of the $23 \mathrm{~S}$ rRNA and the head domain could interact with helix 98, whereas in conformation $2(72,968$ particles, average resolution $8.3 \AA)$ the head domain seems to contact helix 19 of the 23S rRNA. In this context it is noteworthy that helix 24 is in close proximity to the ribosomal protein uL22, which inserts a loop into the ribosomal tunnel exit and thereby could sense the growing nascent chain before it emerges from the tunnel. The lower average resolution of the cryo-EM reconstruction 
with the longer nascent chain did not allow for a detailed analysis of a possible conformational change of the $\alpha$-helices 1 and 2 in the RBD of TF. Taken together, using a known TF substrate as nascent chain we observed that the flexibility of $\mathrm{TF}$ on the translating ribosome decreases with increasing length of the emerging polypeptide chain.

\section{Molecular dynamics simulations of the RNC-bound TF}

The EM and thermophoresis analyses show that RNC-bound TF exhibits a high degree of flexibility, yet the molecular basis of this flexibility remains elusive. Molecular dynamics simulations, capable of describing such dynamic behavior at the atomic level, are thus ideal tools for revealing the cause of TF flexibility. However, the application of simulations to the TF-RNC system has been hampered by the lack of an atomic-resolution structural model of a translating RNC-TF system. Using our cryo-EM data, an atomic model of the ribosometrigger factor-nascent chain complex was built by applying the molecular dynamics flexible fitting (MDFF) method $[28,29]$ to EM data of the TF-GatD85RNC complex (see supplementary figure 5). The cross-correlation coefficient between the atomic model and the EM map was calculated, showing an improvement of quality of fit from 0.75 to 0.82 after the fitting.

Two sub-systems that were studied in our experiments were constructed for simulations (see Methods), one with a nascent GatD chain of 85 amino acids and one with a chain of 145 amino acids length. Here the same amino acid sequences were used for the nascent chains as for the Cryo-EM samples (for details see supplementary table 1). Due to the extensive sampling required for such mobile system, all-atom MD simulations of the system would demand huge computational resources (see Methods). Previous MD studies of a ribosomal-TF system employed a coarse-grained (CG) approach that can significantly accelerate simulations and provided insights into nascent chain folding within ribosome-TF complexes[30-32]. Nevertheless, this approach usually loses the atomic details of proteins and often needs to restrain proteins to their native structures when probing protein dynamics. Alternatively to the CG approach, the PACE (Protein in Atomistic details coupled with Coarse-grained Environment), 
which combines an atomic description of proteins with CG representation of environment, was employed in the present study. PACE has been successfully applied in modeling protein folding and aggregation[33-37]. In our simulations, the $\mathrm{TF}$, the nascent chain and the ribosomal proteins were modeled at an atomic level while the remainder of the system was coarse-grained.

To explore the conformational dynamics of the bound $\mathrm{TF}$, first a 1.2- $\mu \mathrm{s}$ simulation was performed using the short chain model. During the simulation the head and the C-terminal domains of TF fluctuated above the ribosome until the head domain landed on the ribosome. Then the head domain remained bound for the rest of the simulation. We thus performed another three 1.2- $\mu \mathrm{s}$ simulations, each of which ended up with different stable binding poses of the head domain. The four independent simulations revealed that the bound TF is highly flexible above the ribosome surface and lands its head domain over a wide area of the ribosome surface, and that the landing of the head domain arises on a $\mu$ s timescale while the departure should occur on a much longer timescale beyond the capability of the presented simulation approach.

To probe the effect of length of the nascent chain on TF dynamics, another four 1.2- $\mu$ s simulations of TF-ribosome complexes containing a longer nascent chain (145-AA) were also performed. Comparison between the short and long chain simulations will be discussed in the following sections.

\section{Inter-domain motions of TF via pivot points.}

Multiple microseconds of simulations allow us to characterize robustly the collective motions of $\mathrm{TF}$ when bound to the RNC by performing principal component analysis (PCA) with the ProDy software [38]. To explore the flexible motions of TF before its head domain lands on the ribosome surface, only the first $500 \mathrm{~ns}$ of the simulations were analyzed by PCA. The short chain simulations showed that the two most dominant modes of collective motions involve the relative movements between the RBD and the other two domains of TF (Supplementary Fig. 6) via the first pivot point (residue 111 to 121). The third mode of the collective motions captures a relative motion between the $\mathrm{C}$ terminal and the head domain (Supplementary Fig. 6) via the second pivot point (residue 149 to 154 and residue 241 to 248). Similar inter-domain motions via 
the two pivot points were also observed in the simulations involving the longer nascent chain (Gat145), as revealed by the first two PCA modes (Supplementary Fig. 6). These characteristic inter-domain motions via pivot points agree with those reported in prior all-atom MD simulations of the TF monomer in solution $[39,40]$.

These previous all-atom MD simulations of TF monomer in solution revealed that the head and the arm domains undergo a hydrophobic collapse driven by hydrophobic contacts between exposed hydrophobic patches of the two domains. In contrast, such a collapse was not observed in the present study, which is because the nascent peptide shields the hydrophobic patches and prevents the interactions between the two domains (Fig. 5a,b). Taken together, these simulation results suggest that although inter-domain motions of $\mathrm{TF}$ are not affected by the binding of TF to other partners, certain dynamic motions of TF such as hydrophobic collapse can indeed be modulated upon the binding of $\mathrm{TF}$ to the nascent chain.

\section{Conformations of TF bound with a short and long nascent chain.}

Despite the similar collective motions observed both in the short and long chain simulations, distinct conformational features depending on the length of the nascent chains are discernable in the two sets of simulations. In the short chain simulations, the head domain of the TF binds to the ribosome surface (Fig. 4a and c) within a microsecond. The $\mathrm{TF}$ spans the ribosome surface with widespread, extended conformations. The wide range of binding conformations adopted by the ribosome-bound $\mathrm{TF}$ in simulations agrees with our EM experiments where TF bound to the GatD85-RNCs is poorly resolved beyond the RBD due to its conformational variability. In contrast, TF adopts a more compact conformation in the long chain simulations (Fig. $4 \mathrm{~b}$ and d). To further quantify the structural difference of the TF, the following analyses were performed: for each set of simulations (short chain and long chain), we collected all $\mathrm{TF}$ structures sampled during the last $200 \mathrm{~ns}$ of simulations, during which TF in all simulations were bound stably on the ribosome surface, (altogether $800 \mathrm{~ns}$ from four trajectories) and determined the radius of gyration for all backbone atoms 
in the head domain of these TF structures. This radius of gyration measures the spread of the TF head domain on the ribosome surface and was found to be 40.1 $\AA$ and $34.8 \AA$ for the short chain and the long chain simulations, respectively, indicating a more concentrated distribution of $\mathrm{TF}$ conformations when $\mathrm{TF}$ is bound to the longer nascent chain. This result agrees with our experimental observation that TF bound with the long nascent chain is better resolved in EM experiments.

\section{Interactions between TF and the nascent peptide}

To elucidate the molecular origin of the structural difference of the TF bound with nascent chains varying in length, the contacts between $\mathrm{TF}$ and nascent chain were analyzed. In case of the short chain simulations, the GatD85 nascent chain and the head domain of TF show, on average, 7.9 molecular contacts (Fig. 5a and supplementary table 3). In contrast, in case of the long chain simulations, the N-terminus of the chain forms a "collapsed coil" structure that establishes extensive contacts (on average 31.0 contacts) with the head domain (Fig. $5 \mathrm{~b}$ and supplementary table 3), indicating a stronger affinity of the long nascent chain to the $\mathrm{TF}$, which is consistent with microscale thermophoresis experiments. Due to the extensive interactions between the long nascent chain and the $\mathrm{TF}$, the motions of the TF head and C-terminal domains are significantly restricted. Such restriction explains why in the long chain simulations the TF is conformational less widespread on the ribosome surfaces.

To further examine the detailed interactions formed between $\mathrm{TF}$ and nascent chain, various types of interactions between the nascent peptide and TF in the simulations were monitored. The RBD of the TF interacts with the nascent peptide mainly through hydrophobic interactions (Fig. 5c and supplementary tables 4 and 5), agreeing with the known hydrophobic binding motif in the RBD [41]. Hydrophobic interactions still dominate between the C-terminal domain and the nascent peptide, but more hydrophilic interactions arise (Fig. 5c and supplementary tables 6 and 7), showing that the TF can interact with, and hence offer protection to, nascent peptides of various hydrophobicity[18]. For the head domain, only a few residues are found interacting with the nascent peptide in the TF-GatD85-RNC simulations (supplementary table 8), while many more residues 
of the head domain interact with the GatD145 nascent chain through both hydrophobic and hydrophilic interactions (Fig. 5c and supplementary table 9). A recent NMR study of TF-PhoA complex in solution has reported four major hydrophobic binding sites in TF [7]. Comparison between the simulations and the NMR results shows an overall agreement in terms of the binding sites on the C-terminal and the head domain. More than half of the hydrophobic residues in the reported binding sites (28 out of 49) on these domains were also found to frequently interact with the nascent chain in the simulations (Supplementary table 6-9). The NMR experiment and the simulation agree less well for the RBD domain: the simulations identified considerable hydrophobic interactions (Supplementary table 4,5) with the nascent chain, which were not found in the NMR experiment. The difference can be attributed to a structural rearrangement of the RBD upon binding to the ribosome as observed in our cryo-EM experiments, which expose the hydrophobic surface of the RBD to the nascent chain (see Discussion). Moreover, compared to the NMR experiment that was done in the absence of ribosomes, the local concentration of the nascent chain near the RBD is much higher in case of the ribosome-TF-GatD complex.

\section{Discussion:}

The molecular chaperone TF is the only chaperone in bacteria that directly binds to the ribosome in order to engage the emerging nascent chain at the tunnel exit. Our cryo-EM structure of a TF-GatD85-RNC shows the RBD of TF essentially in the same position as observed in a previous structure of lower resolution. The RBD contacts the ribosome at the ribosomal proteins uL23 and uL29 in agreement with previously published data $[1,17,18,25]$. Our reconstituted map reveals that the first "landing platform" for a hydrophobic stretch in the nascent chain is localized on the rearranged surface of the RBD of TF. The rearrangement of two helices provides a hydrophobic surface for interaction as was suggested by Schlünzen et al. based on their crystal structure of TF RBD bound to the $D$. radiodurans 50S subunit [17]. The localization of this hydrophobic surface is also in agreement with earlier crosslinking studies where a zero-space crosslinker (Bpa) at position 61 in TF (see Fig. 2c) was the first position to crosslink to a 47 
amino acid long nascent chain of isocitrate dehydrogenase on the ribosome [18]. Notably, a ribosome profiling study characterizing the interactome of $\mathrm{TF}$ concluded that in vivo TF engages ribosomes stable enough for detection only after the nascent chain has reached a length of $\sim 100$ amino acids[19]. This trend is in agreement with our finding that the affinity of TF for RNCs increases fivefold between the short ( 85 amino acids) and the longer construct (145 amino acids). Apparently, the early encounter with its limited interactions between TF and the nascent chain is not sufficient for a stable interaction.

Our data suggest that at this stage the TF molecule itself is very flexible. Since translation speed in bacteria is rather fast (up to 20 aa/s), several processes such as modification of the $\mathrm{N}$-terminus by peptidyl deformylase (PDF) or methionine amino peptidase (MAP) must happen quickly after the polypeptide chain has emerged. A recent study of the Wintermeyer lab addressed the possible orchestration of different targeting, processing and folding factors on the ribosome using FRET [42] and found that neither PDF nor MAP interfered with $\mathrm{TF}$ binding to the ribosome. It is tempting to speculate that the observed flexibility within the TF molecule during this early encounter with the translating ribosome facilitates this concomitant interaction of several ribosome interactors at the limited binding space surrounding the ribosomal tunnel exit.

As the nascent peptide becomes extended it most likely engages in additional interactions with the TF molecule, potentially with the surface between the two arms of the CTD and the surface of the PPIase domain where further hydrophobic patches are localized [1]. Indeed, we observed such interactions in our simulations and they could explain the higher rigidity of TF observed in our second cryo-EM structure of a TF-GatD145-RNC complex where the ribosome harbors a longer nascent chain and the increased affinity of TF for RNCs carrying these longer chains in our thermophoresis experiments. In this more rigid conformation TF could serve as a holdase or even unfoldase of the newly synthesized polypeptide as suggested by Hoffmann et al [43].

In agreement with our interaction sites between TF and the nascent chain a recent elegant NMR study on TF free in solution in complex with its substrate PhoA identified four distinct binding sites within the TF molecule [7]. Three of 
these sites are located in the substrate binding site and one is located in the PPIase domain overlapping with its catalytic site. Interestingly, in solution (not bound to the ribosome) no interaction of the substrate PhoA with the RBD of TF was observed and the two $\alpha$ helices of the RBD in the NMR structure do not show such a rearrangement as observed in our cryo-EM structure of the ribosome bound TF. This indicates that binding of TF to the ribosome may indeed be required to prime the RBD for interaction. The rearrangement of the two helices of the RBD would lead to the presentation of the hydrophobic landing surface as observed in our cryo-EM reconstruction of a TF-GatD85-RNC complex. This first encounter at the RBD could direct the growing nascent chain towards the further downstream lying substrate binding sites on the CTD and in the end towards the PPIase domain [7].

Eventually, TF leaves the ribosome while staying bound to the nascent peptide, allowing the next $\mathrm{TF}$ molecule to be recruited to the ribosome to protect the elongating chain [5]. Moreover, the length of the nascent peptide has been shown to not only modulate the association but also the dissociation of TF from the ribosome [44]. Interestingly, our simulations suggest that, as the nascent peptide grows longer, trigger factor can be rigidified (supplemental table 2) by the molecular interactions between the head domain and the longer nascent peptide (Fig. 5b). This may also facilitate the dissociation of TF from the ribosome. However, as the dissociation of TF is usually very slow and cannot be directly observed through our simulations, it remains an open question whether the rigidification could correlate with the TF dissociation. Furthermore, a longer nascent peptide is capable of forming more extensive interactions with the TF as shown in the simulations. They could serve to keep the TF bound to the nascent chain after it leaves the ribosome.

Taken together TF initially encounters the emerging nascent peptide chain on the ribosome as a rather flexible molecule offering a first hydrophobic binding platform upon subtle rearrangement of its RBD (see Fig. 6). It gains rigidity as the nascent chain grows longer in order to eventually dissociate from the ribosome while remaining bound to the emerging peptide as a foldase or holdase. 


\section{Materials and Methods:}

\section{Purification of TF:}

TF and TF150C carrying a N-terminal His6-tag followed by a Tobacco Etch Virus (TEV) protease cleavage site was expressed from a pPROEX-HTa based plasmid (gift of F.U. Hartl) [5] in E. coli ER2566 (New England Biolabs). TF was purified by immobilized metal chelate affinity chromatography using Ni-NTA agarose beads (Qiagen), followed by removal of the His-tag by TEV protease cleavage. Uncleaved TF and TEV protease (also carrying a His6-tag) were removed by an additional passage over Ni-NTA agarose. Further purification via anion exchange chromatography (POROS HQ20) and a final size exclusion chromatography (Superdex S200, GE healthcare) resulted in a homogeneous pool of untagged TF.

\section{Plasmids:}

Coding sequences for GatD-TnaC nascent chains (85AA or 145AA) used in this study were cloned into pBAD vectors (Invitrogen). For Sequence details see supplementary table 1 .

\section{In vivo preparation of TnaC stalled GatD-RNCs:}

Plasmids were freshly transformed into E. coli KC6 ( $\Delta$ smpB, $\Delta$ ssrA) [21]. Cells were grown at $37^{\circ} \mathrm{C}$ on LBAmp medium to an $\mathrm{OD}_{600}$ of 0.6 . Expression of the stalling peptide was induced by addition of $0.2 \%(\mathrm{w} / \mathrm{v})$ arabinose and growth was continued for $1 \mathrm{~h}$. Cells were pelleted and resuspended in $1 \mathrm{ml}$ per gram wet cells of buffer ${ }^{250}$ (50 mM HEPES pH 7.2; 250 mM KOAc; 25 mM Mg(OAc) $2 ; 250$ $\mathrm{mM}$ sucrose; 0,1 \% DDM; $1 \mathrm{mM}$ tryptophane, protease inhibitor (Roche), $34 \mu \mathrm{g} / \mathrm{ml}$ chloramphenicol). Cells were disrupted by three passages through a microfluidizer at $17 \mathrm{kpsi}$. The cell lysate was cleared $(25 \mathrm{~min}$ at $16,000 \mathrm{rpm}$ at $+4^{\circ} \mathrm{C}$ in a SS34 rotor) and ribosomes were pelleted from the SS34 supernatant (Ti45, 2h, 42,000 $\mathrm{rpm}, 4^{\circ} \mathrm{C}$ ). Pelleted ribosomes were resuspended in $2 \mathrm{ml}$ buffer ${ }^{250}$ per liter of original culture. RNCs were bound to $300 \mu \mathrm{l}$ of a $\mathrm{Co}^{2+}$ chelating resin (Talon, Clontech) per liter original culture $\left(1 \mathrm{~h},+4^{\circ} \mathrm{C}\right)$, washed with 10 column volumes of buffer ${ }^{250}$, followed by 2 column volumes of buffer500 (same as buffer 250 but with $500 \mathrm{mM} \mathrm{KOAc}$ ) and eluted with 3 column volumes 
of buffer ${ }^{250}$ supplemented with $100 \mathrm{mM}$ imidazol. RNCs were loaded onto a 10$40 \%$ sucrose gradient (in $50 \mathrm{mM}$ HEPES pH 7.2; $25 \mathrm{mM} \mathrm{Mg(OAc)} 2 ; 50 \mathrm{mM} \mathrm{KCl}$; $10 \mathrm{mM} \mathrm{NH}_{4} \mathrm{Cl}$ ), spun for $3 \mathrm{~h}$ at $40,000 \mathrm{rpm}$ and $+4^{\circ} \mathrm{C}$ in a SW40 rotor (Beckman) and fractionated using a gradient station (Biocomp Instruments). RNC containing fractions were pooled, RNCs were pelleted (TLA110, $4 \mathrm{~h}, 40,000 \mathrm{rpm},+4^{\circ} \mathrm{C}$ ) and resuspended in $150 \mu \mathrm{l}$ of buffer ${ }^{250}$ per liter of starting culture. The quality of the RNCs was assessed by Western blotting using an HA antibody.

\section{TF-GatD-RNCs reconstitution:}

GatD-RNCs (80 nM) were incubated with a 10-fold molar excess of TF (800 nM) in 20 mM HEPES, pH 7.4; $100 \mathrm{mM} \mathrm{KOAc;} 10 \mathrm{mM} \mathrm{Mg(OAc)}$; 2 mM DTT in a final volume of $20 \mu \mathrm{l}$ for $10 \mathrm{~min}$ at room temperature. The samples were placed on ice and directly subjected to vitro botting.

\section{Electron microscopy and image processing:}

Freshly prepared sample was applied to 2nm pre-coated Quantifoil R3/3 holey carbon supported grids and vitrified using a Vitrobot Mark IV (FEI Company). TF-GatD85-RNC samples were visualized on a Tecnai F30 field emission gun electron microscope under low dose conditions (20 e- per $\AA^{2}$ ) at $300 \mathrm{keV}$ (Ultra Structure Network, Berlin) applying defocus values from $-1.5 \mu \mathrm{m}$ to $-3.5 \mu \mathrm{m}$, nominal magnification 39,000 x. Micrographs were scanned on a Heidelberg drum scanner resulting in a pixel size of $1.2375 \AA$.

TF-GatD145-RNC samples were visualized on a Titan Krios TEM (FEI Company) under low-dose conditions (20 e- per $\AA^{2}$ ) at $200 \mathrm{keV}$ applying defocus values from $-1.2 \mu \mathrm{m}$ to $-4.0 \mu \mathrm{m}$ at a magnification of $148,721 \mathrm{x}$ at the plane of CCD using a TemCam-F416 CMOS camera (TVIPS GmbH, 4,096 x 4,096 pixel, $15.6 \mu \mathrm{m}$ pixel, $1 \mathrm{~s} /$ full frame), resulting in an image pixel size of $1.0489 \AA$ (object scale).

Data collection was facilitated by the semi-automated software EM-TOOLS (TVIPS GmbH), allowing manual selection of appropriate grid meshes and holes in the holey carbon film. The acquisition automatically performed a re-centering, drift and focus correction before the final spot scan series were taken. Long-term TEM instabilities in beam shift, astigmatism and coma were corrected by 
EMTOOLS regularly (for example, every $45 \mathrm{~min}$ ). Selected on the basis of power spectra quality, typically $70 \%$ of the recorded images were used for the subsequent reconstruction. Data processing was essentially done as described before [23].

Both datasets behaved similarly. All ribosomal particles contained P-site tRNA. The dataset was split according to the conformation of the E-site stalk (L1 stalk). Short NC chain E-site stalk in outside conformation. Focused sorting for presence / absence of ligand (TF) was essentially done as described in Leidig et al. [45]. In the case of the long nascent chain the ligand containing dataset was split into two final populations exhibiting distinct conformations of the TF head domain.

\section{Microscale thermophoresis (MST):}

TF carrying a unique cysteine at position 150 was purified and the cysteine was labelled with Alexa Fluor 568 C5 maleimide (life technologies) according to the manufacturer's protocols. Labelling efficiency was determined via molar extinction coefficients. GatD-RNCs ( 1 to $3200 \mathrm{nM}$ ) were titrated against labelled TF (20 nM) in MST-buffer (20 mM HEPES, pH 7.4; $100 \mathrm{mM} \mathrm{KOAc,} 10 \mathrm{mM}$ $\mathrm{Mg}(\mathrm{OAc})$; 2 mM DTT; $1 \mu \mathrm{M}$ BSA; $0.01 \%$ Tween 20). RNCs carried either the same short or long GatD nascent polypeptide as used for structure determination. Thermophoresis was monitored in a Monolith NT-115 instrument (blue/green) using hydrophilic micro capillaries (NanoTemper Technologies, Munich, Germany) with the following settings: LED intensity adjusted to yield 100 fluorescence counts; IR-laser was set to $2.0 \mathrm{~V} / 80 \%$ intensity, sample was heated until an equilibrium was reached $(\sim 100 \mathrm{~s})$.

\section{MDFF modeling:}

The atomic model of the ribosome-trigger factor-nascent chain complex was built in steps. First, an atomic structure of the ribosome (PDB ID: 2WWQ2WWL)[21] was flexibly fit into the ribosomal densities of the $7.7 \AA$ EM map of the TF-GatD85-RNC complex by the molecular dynamics flexible fitting (MDFF) method $[28,29]$. Second, the structure of the nascent chain inside the exit tunnel was modeled with HHpred [46], and then refined by MDFF simulation with a positionally constrained ribosome. Third, TF was modeled starting from a crystal 
structure of TF factor (PDB ID: 1W26)[1], with helix 1 and 2 of the RBD rearranged by Coot as described in Results. The RBD, the C-terminal domain and the head domain were then fitted into three EM maps of the TF-GatD85-RNC complex, resolved at $7.7 \AA, 16.5 \AA$ and $35 \AA$ resolution, respectively, in one MDFF simulation (Supplementary Fig. 4). Each domain was coupled only to the respective EM map in the MDFF simulation. Since the C-terminal and the head domain were fitted to maps of lower resolution, the secondary structure restraints [28] applied to the C-terminal domain were twice stronger than default, and domain restraints, which keep the backbone structure rigid during fitting, were applied to the head domain. This way of performing MDFF, namely coupling various domains to maps of different resolutions with different level of structural restraints, permits EM data at lower resolution to be employed for flexible fitting. To improve the quality of the fitted models, a better solvent description, compared to that in in-vacuo fitting [28], was furnished by the generalized Born implicit solvent model [47] in NAMD [48] during all the MDFF simulations. All MDFF simulations were performed for $5 \mathrm{~ns}$, using the same set of parameters as employed in [47].

\section{Simulated systems and molecular dynamics protocols:}

To carry out the hybrid simulations, the nascent chain needs to be extended beyond the exit tunnel. Without well-defined EM densities for the nascent chain outside the ribosome, an initial model of the 85-AA nascent peptide was built by placing amino acids inside the cradle of the trigger factor according to distance information from cross-linking experiments [18]. The chain was then subjected to $50 \mathrm{~ns}$ of all-atom equilibration simulations in implicit solvent [49], with the TF and the ribosome constrained in positions and with harmonic distance restraints applied between the nascent chain and the trigger factor; the distance restraints were set up based on the cross-linking experiments [18] (Supplementary table 10). To extend the chain to 145 amino acids, the model of the 85-AA chain was mutated and 60 more amino acids were added in a straight conformation to the $\mathrm{N}$-terminus, followed by $50 \mathrm{~ns}$ of all-atom equilibration simulations in implicit solvent [49]. Position and distance restraints similar to those applied in the equilibration of the 85-AA chain were employed. 
The two atomic models were cut smaller to reduce the simulations size by retaining only molecular structures that are within $80 \AA$ of the trigger factor. Ribosome-bound $\mathrm{Mg}^{2+}$ ions were added by the cionize [50] plugin of VMD [51]. A solvated all-atom representation of such sub-system involves 1.3 million atoms. To perform multiple microsecond-long simulations as done in the present study using all-atom representation would require over 384 million core-hours on the Stampede supercomputer. To alleviate such huge computational burden, the subsystems were instead coarse-grained and described at hybrid resolutions using the PACE force field $[33,35,52]$. This force field, which was developed as a complement to the MARTINI force field [53], describes the solvent environment at a coarse-grained level while representing proteins at a united-atom level, allowing efficient long-time simulations of folding of several proteins into their native structures $[33,34,37]$. In the present study, the ribosomal proteins, the trigger factor and the nascent peptide were modeled at a united-atom level. The ribosomal RNAs were described with a coarse-grained RNA model [54] developed recently on the basis of the MARTINI force field. The rest of the systems, including water and ions, were modeled by means of the MARTINI force field. Each system was solvated in a 220 × 220 × $220 \AA^{3}$ water box and buffered at $0.1 \mathrm{M} \mathrm{Na}^{+} \mathrm{Cl}^{-}$concentration. All simulations were performed in NPT ensemble using a version of NAMD 2.9 [48] which supports the PACE force field. The following parameters were employed in the simulations: temperature was maintained at $310 \mathrm{~K}$ using Langevin dynamics with a damping constant of 0.2 $\mathrm{ps}^{-1}$; pressure was maintained at 1 atm using Nosè-Hoover Langevin piston pressure control with an oscillation time of $100 \mathrm{fs}$ and a decay time of $50 \mathrm{fs}$. The RESPA multiple-time-stepping algorithm [55, 56] was adopted with a 4-fs integration time step, short-range forces calculated every time step, and longrange electrostatics evaluated every time step. During the simulations, the ribosomal RNA beads and the ribosome-bound $\mathrm{Mg}^{2+}$ ions were fixed in positions, which was justified as such constraints have been shown to have only a small effect on simulated nascent chain dynamics [30-32]. 


\section{Accession numbers:}

Cryo-electron microscopy maps for 70S TF-GatD85-RNC complex and the 70S TF-GatD145-RNC complex conformation 1 and 2 have been deposited in the EMDataBank with the accession codes EMD-2695, EMD-2696 and EMD-2711 respectively. The respective coordinates for the electron-microscopy-based model are deposited in the Protein Data Bank (ㄴurd for the RBD of TF).

\section{Acknowledgments:}

We would like to thank F.U. Hartl for the TF expression plasmid and C. Ungewickell for assistance with cryo-EM data collection. This work was supported by grants of the Deutsche Forschungsgemeinschaft, SFB594 (to R.B. and B.B.), CIPSM (to R.B.), FOR967 (to R.B.), an advanced investigator grant of the ERC CRYOTRANSLATION (to R.B.), a scholarship of the IMPRIS-LS to J.D. and the Genecenter cryo-EM facility. This work was also supported by grants from the National Institutes of Health (9P41GM104601) and the National Science Foundation (PHY0822613). Computer time on Stampede at the Texas Advanced Computing Center (TACC) was provided by grant MCA93S028 from the Extreme Science and Engineering Discovery Environment (XSEDE), which is supported by National Science Foundation grant number OCI-1053575. This research is also part of the Blue Waters Early Science project, which is supported by the National Science Foundation (award number OCI 07-25070) and the state of Illinois. MD simulations in this work were performed using NAMD [48], and molecular images in this article were rendered using VMD [51]. 


\section{Author Contributions:}

R.B. and B.B. conceived the structural study, J.D. performed the sample preparation, processed the cryo-EM data and built the molecular models, E.v.d.S. established protocols for generation of in vivo assembled RNCs, O.B. performed cryo-EM data collection, K.S. and J.G. conceived the simulation study, K.Y.C. and W.H. performed the simulations. R.B., B.B., J.D., K.S. and K.Y.C. interpreted the results and wrote the manuscript. 


\section{Figure legends:}

Figure 1: Cryo-EM reconstruction of a 70S TF-GatD85-RNC complex. (a) Schematic representation of the RNC used for reconstituting the complex. (b) Cryo-EM reconstruction of the 70S TF-GatD85-RNC complex. Bottom view. The ribosomal 30S subunit is shown in yellow, the 50S subunit in grey and trigger factor (TF) in solid red (7.7 $\AA$ resolution) or as red mesh filtered at lower resolution (16.5 A). (c) Cryo-EM reconstruction of the 70S TF-GatD85-RNC complex. Side view. Colors as in $\mathbf{b}$ but with the peptidyl-tRNA shown in green. (d) Density map as in c, but the 50S subunit is cut along the polypeptide exit tunnel for visualization of the nascent chain (NC).

Figure 2: Structural rearrangement of the TF ribosome binding domain. (a) Isolated density for the peptidyl-tRNA with the color code schematically represented on the left side. (b) A crystal structure of E. coli TF RBD in solution (PDB ID: 10MS) [25] was fitted into the EM density and regularized using the Coot algorithm [26]. The electron density is represented as a red mesh with the ribbon representation for the fitted model in solid red in the left panel. The middle panel shows a comparison of the crystal structure of TF RBD in solution (PDB ID: 10MS, blue) with the TF RBD model fitted to the EM density (red). The right panel shows the same comparison after rotation by $45^{\circ}$ around the $y$-axis. (c) Left panel: molecular model of the 50S subunit (pdb 2WWQ)[21] with electron density for TF RBD (red mesh), hydrophobic stretch (yellow) and alpha helix (green) of GatD NC and the fitted model as red ribbon representation. The green spheres indicate published crosslinks of TF to short nascent chains as they emerge [18]. Right panel same as in left panel but with surface hydrophobicity (yellow) representation for the fitted TF RBD. For better visualization the hydrophobic stretch is shown as orange mesh in this panel. (d) Surface hydrophobicity representation for the tip of the RBD of TF which is shown in the same orientation as in (c). Comparison of the hydrophobic surface of ribosome bound TF RBD model fitted to the EM density (with the rearranged helices 1 and 2 )(top) with the hydrophobic surface of TF RBD crystal structure in solution 
(PDB ID: 10MS, bottom). Hydrophobic surface is depicted in yellow, the remainder of the surface is shown in grey.

Figure 3: Cryo-EM reconstruction of a 70S TF-GatD145-RNC complex with a longer nascent chain. (a) Schematic representation of the RNC used for reconstituting the complex. (b) Affinity of TF for GatD-RNCs of different length determined by microscale thermophoresis. GatD-RNCs were titrated against TF150C-Alexa 568. The red curve represents RNCs with the shorter nascent chain (GatD85), whereas the blue curve represents RNCs with the longer nascent chain (GatD145). (c) Cryo-EM reconstruction of the 70S-GatD-RNC-TF complex carrying the shorter (left) or the longer (middle and right) NC (filtered at $13.1 \AA$ for clarity). The upper panel depicts bottom views of the ribosome. The ribosomal 30S subunit is shown in yellow, the 50S subunit in grey and trigger factor (TF) in red. The middle panel shows side views of the same complexes. The lower panel depicts a front view onto the PPIase domain of TF of the two different conformations observed in the TF-GatD145-RNC complex. A molecular model (PDB ID: 2WWQ) [21] of the 50S subunit is fitted as a ribbon representation into the electron density map shown in grey. Possible contacts of TF with the ribosomal 50S subunit are indicated.

Figure 4: Average conformation of the trigger factor in the last $200 \mathrm{~ns}$ of the TFGatD85-RNC simulations ( $a$ and $c$ ) and the TF-GatD145-RNC simulations (b and d). Figure $a$ and $b$ show the side view and figure $c$ and d show the bottom view of the system. TF is colored blue, red, orange and yellow, respectively for each of the four simulations, with molecular surface of the head domain shown for clarity. The ribosome is colored grey. The trigger factor adopts a more compact conformation (comparing a with b and c with d) in the 145-AA chain simulations. Only ribosomal structures that are within $80 \AA$ of the trigger factor were included in the simulations and were shown in the figures.

Figure 5: (a) Average conformation of the N-terminus of the GatD85 nascent peptide in the last 200 ns of the short chain simulations. The nascent peptide is 
colored green and the trigger factor is colored red for the head domain and grey for the C-terminal domain. The N-terminus of the nascent chain contacts slightly with, or even detaches from, the head domain of the trigger factor. (b) Average conformation of the N-terminus of the GatD145 nascent peptide in the last $200 \mathrm{~ns}$ of the long chain simulations. The same coloring scheme as in (a) is employed. The N-terminus of the GatD145 nascent chain forms a "collapsed coil" structure which contacts extensively with the head domain of the trigger factor. (c) Amino acid residues of TF in the RBD (left), the C-terminal domain (middle) and the head domain (right) that interact with the nascent peptide in the simulations are shown. These residues are shown with a VdW representation according to the following coloring scheme: residues that participate in hydrophobic interactions with the chain are colored yellow while residues that interact through hydrophilic interactions are colored blue, red and green for positively charged, negatively charged and neutral amino acids, respectively.

Figure 6: Model illustrating the dynamic behavior of trigger factor on the ribosome. The colour scheme is as follows: large ribosomal subunit (grey) emerging nascent chain (green), ribosomal proteins involved in trigger factor binding (cyan and bright green), TF RBD (red), TF C-terminal domain (orange), TF head domain (yellow). Flexibility is illustrated by the fading out "shadows". The rearranged TF RBD is labelled by an asterisk.

\section{References:}

[1] Ferbitz L, Maier T, Patzelt H, Bukau B, Deuerling E, Ban N. Trigger factor in complex with the ribosome forms a molecular cradle for nascent proteins. Nature. 2004;431:590-6. 
[2] Genevaux P, Keppel F, Schwager F, Langendijk-Genevaux PS, Hartl FU, Georgopoulos C. In vivo analysis of the overlapping functions of DnaK and trigger factor. EMBO reports. 2004;5:195-200.

[3] Merz F, Hoffmann A, Rutkowska A, Zachmann-Brand B, Bukau B, Deuerling E. The $\mathrm{C}$-terminal domain of Escherichia coli trigger factor represents the central module of its chaperone activity. The Journal of biological chemistry. 2006;281:31963-71.

[4] Gupta R, Lakshmipathy SK, Chang HC, Etchells SA, Hartl FU. Trigger factor lacking the PPIase domain can enhance the folding of eukaryotic multi-domain proteins in Escherichia coli. FEBS letters. 2010;584:3620-4.

[5] Kaiser CM, Chang HC, Agashe VR, Lakshmipathy SK, Etchells SA, Hayer-Hartl $\mathrm{M}$, et al. Real-time observation of trigger factor function on translating ribosomes. Nature. 2006;444:455-60.

[6] Lakshmipathy SK, Gupta R, Pinkert S, Etchells SA, Hartl FU. Versatility of trigger factor interactions with ribosome-nascent chain complexes. The Journal of biological chemistry. 2010;285:27911-23.

[7] Saio T, Guan X, Rossi P, Economou A, Kalodimos CG. Structural basis for protein antiaggregation activity of the trigger factor chaperone. Science. 2014;344:1250494.

[8] Woolhead CA, McCormick PJ, Johnson AE. Nascent membrane and secretory proteins differ in FRET-detected folding far inside the ribosome and in their exposure to ribosomal proteins. Cell. 2004;116:725-36.

[9] Lu J, Deutsch C. Folding zones inside the ribosomal exit tunnel. Nature structural \& molecular biology. 2005;12:1123-9.

[10] Bhushan S, Gartmann M, Halic M, Armache JP, Jarasch A, Mielke T, et al. alpha-Helical nascent polypeptide chains visualized within distinct regions of the ribosomal exit tunnel. Nature structural \& molecular biology. 2010;17:313-7.

[11] Wilson DN, Beckmann R. The ribosomal tunnel as a functional environment for nascent polypeptide folding and translational stalling. Current opinion in structural biology. 2011;21:274-82.

[12] Holtkamp W, Kokic G, Jager M, Mittelstaet J, Komar AA, Rodnina MV.

Cotranslational protein folding on the ribosome monitored in real time. Science.

2015;350:1104-7.

[13] Nilsson OB, Hedman R, Marino J, Wickles S, Bischoff L, Johansson M, et al. Cotranslational Protein Folding inside the Ribosome Exit Tunnel. Cell Rep.

2015;12:1533-40.

[14] Cabrita LD, Cassaignau AM, Launay HM, Waudby CA, Wlodarski T, Camilloni C, et al. A structural ensemble of a ribosome-nascent chain complex during cotranslational protein folding. Nature structural \& molecular biology.

2016;23:278-85.

[15] Marino J, von Heijne G, Beckmann R. Small protein domains fold inside the ribosome exit tunnel. FEBS letters. 2016;590:655-60.

[16] Baram D, Pyetan E, Sittner A, Auerbach-Nevo T, Bashan A, Yonath A. Structure of trigger factor binding domain in biologically homologous complex with eubacterial ribosome reveals its chaperone action. Proceedings of the National Academy of Sciences of the United States of America. 2005;102:1201722. 
[17] Schlunzen F, Wilson DN, Tian P, Harms JM, McInnes SJ, Hansen HA, et al. The binding mode of the trigger factor on the ribosome: implications for protein folding and SRP interaction. Structure. 2005;13:1685-94.

[18] Merz F, Boehringer D, Schaffitzel C, Preissler S, Hoffmann A, Maier T, et al. Molecular mechanism and structure of Trigger Factor bound to the translating ribosome. The EMBO journal. 2008;27:1622-32.

[19] Oh E, Becker AH, Sandikci A, Huber D, Chaba R, Gloge F, et al. Selective ribosome profiling reveals the cotranslational chaperone action of trigger factor in vivo. Cell. 2011;147:1295-308.

[20] Gong F, Yanofsky C. Instruction of translating ribosome by nascent peptide. Science. 2002;297:1864-7.

[21] Seidelt B, Innis CA, Wilson DN, Gartmann M, Armache JP, Villa E, et al. Structural insight into nascent polypeptide chain-mediated translational stalling. Science. 2009;326:1412-5.

[22] Bischoff L, Wickles S, Berninghausen O, van der Sluis EO, Beckmann R. Visualization of a polytopic membrane protein during SecY-mediated membrane insertion. Nature communications. 2014;5:4103.

[23] Becker T, Franckenberg S, Wickles S, Shoemaker CJ, Anger AM, Armache JP, et al. Structural basis of highly conserved ribosome recycling in eukaryotes and archaea. Nature. 2012;482:501-6.

[24] Benavente R, Esteban-Torres M, Kohring GW, Cortes-Cabrera A, SanchezMurcia PA, Gago F, et al. Enantioselective oxidation of galactitol 1-phosphate by galactitol-1-phosphate 5-dehydrogenase from Escherichia coli. Acta crystallographica Section D, Biological crystallography. 2015;71:1540-54.

[25] Kristensen 0, Gajhede M. Chaperone binding at the ribosomal exit tunnel. Structure. 2003;11:1547-56.

[26] Emsley P, Lohkamp B, Scott WG, Cowtan K. Features and development of Coot. Acta crystallographica Section D, Biological crystallography. 2010;66:486501.

[27] Seidel SA, Dijkman PM, Lea WA, van den Bogaart G, Jerabek-Willemsen M, Lazic A, et al. Microscale thermophoresis quantifies biomolecular interactions under previously challenging conditions. Methods. 2013;59:301-15.

[28] Trabuco LG, Villa E, Mitra K, Frank J, Schulten K. Flexible Fitting of Atomic Structures into Electron Microscopy Maps Using Molecular Dynamics. Structure. 2008;16:673-83.

[29] Trabuco LG, Villa E, Schreiner E, Harrison CB, Schulten K. Molecular Dynamics Flexible Fitting: A practical guide to combine cryo-electron microscopy and X-ray crystallography. Methods. 2009;49:174-80.

[30] O'Brien EP, Christodoulou J, Vendruscolo M, Dobson CM. New Scenarios of Protein Folding Can Occur on the Ribosome. Journal of the American Chemical Society. 2011;133:513-26.

[31] O'Brien EP, Christodoulou J, Vendruscolo M, Dobson CM. Trigger factor slows co-translational folding through kinetic trapping while sterically protecting the nascent chain from aberrant cytosolic interactions. Journal of the American Chemical Society. 2012;134:10920-32.

[32] O'Brien EP, Hsu SD, Christodoulou J, Vendruscolo M, Dobson CM. Transient tertiary structure formation within the ribosome exit port. Journal of the American Chemical Society. 2010;132:16928-37. 
[33] Han W, Schulten K. Further optimization of a hybrid united-atom and coarse-grained force field for folding simulations: Improved backbone hydration and interactions between charged side chains. Journal of Chemical Theory and Computation. 2012;8:4413-24.

[34] Han W, Schulten K. Characterization of Folding Mechanisms of Trp-cage and WW-domain by Network Analysis of Simulations with a Hybrid-resolution Model. Journal of Physical Chemistry B. 2013;117:13367-77.

[35] Han W, Wan C-K, Jiang F, Wu Y-D. PACE force field for protein simulations. 1. full parameterization of version 1 and verification. Journal of Chemical Theory and Computation. 2010;6:3373-89.

[36] Han W, Wan C-K, Wu Y-D. Toward a coarse-grained model coupled with a coarse-grained solvent model: solvation free energies of amino acid side chain. Journal of Chemical Theory and Computation. 2008;4:1891-901.

[37] Han W, Wan C-K, Wu Y-D. PACE force field for protein simulations. 2. folding simulations of peptides. Journal of Chemical Theory and Computation.

2010;6:3390-402.

[38] Bakan A, Meireles LM, Bahar I. ProDy: Protein Dynamics Inferred from Theory and Experiments. Bioinformatics. 2011;27:1575-7.

[39] Thomas AS, Mao S, Elcock AH. Flexibility of the Bacterial Chaperone Trigger Factor in Microsecond-Timescale Molecular Dynamics Simulations. Biophysical Journal. 2013;105:732-44.

[40] Singhal K, Vreede J, Mashaghi A, Tans SJ, Bolhuis PG. Hydrophobic Collapse of Trigger Factor Monomer in Solution. PLoS One. 2013;8:e59683.

[41] Patzelt H, Rüdiger S, Brehmer D, Kramer G, Vorderwülbecke S, Schaffitzel E, et al. Binding specificity of Escherichia coli trigger factor. Proceedings of the National Academy of Sciences, USA. 2001;98:14244-9.

[42] Bornemann T, Holtkamp W, Wintermeyer W. Interplay between trigger factor and other protein biogenesis factors on the ribosome. Nature communications. 2014;5:4180.

[43] Hoffmann A, Becker AH, Zachmann-Brand B, Deuerling E, Bukau B, Kramer

G. Concerted action of the ribosome and the associated chaperone trigger factor confines nascent polypeptide folding. Molecular cell. 2012;48:63-74.

[44] Rutkowska A, Mayer MP, Hoffmann A, Merz F, Zachmann-Brand B, Schaffitzel C, et al. Dynamics of trigger factor interaction with translating ribosomes. Journal of Biological Chemistry. 2008;283:4124-32.

[45] Leidig C, Bange G, Kopp J, Amlacher S, Aravind A, Wickles S, et al. Structural characterization of a eukaryotic chaperone--the ribosome-associated complex. Nature structural \& molecular biology. 2013;20:23-8.

[46] Söding J, Biegert A, Lupas AN. The HHpred interactive server for protein homology detection and structure prediction. Nucleic Acids Research. 2005;33:W244-W8.

[47] Tanner DE, Chan K-Y, Phillips J, Schulten K. Parallel Generalized Born Implicit Solvent Calculations with NAMD. Journal of Chemical Theory and Computation. 2011;7:3635-42.

[48] Phillips JC, Braun R, Wang W, Gumbart J, Tajkhorshid E, Villa E, et al. Scalable Molecular Dynamics with NAMD. Journal of Computational Chemistry. 2005;26:1781-802. 
[49] Tanner DE, Phillips JC, Schulten K. GPU/CPU Algorithm for Generalized Born / Solvent-Accessible Surface Area Implicit Solvent Calculations. Journal of Chemical Theory and Computation. 2012;8:2521-30.

[50] Stone JE, Phillips JC, Freddolino PL, Hardy DJ, Trabuco LG, Schulten K. Accelerating Molecular Modeling Applications with Graphics Processors. Journal of Computational Chemistry. 2007;28:2618-40.

[51] Humphrey W, Dalke A, Schulten K. VMD - Visual Molecular Dynamics. Journal of Molecular Graphics. 1996;14:33-8.

[52] Wan C-K, Han W, Wu Y-D. Parameterization of PACE Force Field for Membrane Environment and Simulation of Helical Peptides and Helix-Helix Association. Journal of Chemical Theory and Computation. 2012;8:300-13. [53] Marrink SJ, Risselada HJ, Yefimov S, Tieleman DP, de Vries AH. The MARTINI force field: coarse grained model for biomolecular simulations. Journal of Physical Chemistry B. 2007;111:7812-24.

[54] Khalid S, Bond PJ, Holyoake J, Hawtin RW, Sansom MSP. DNA and lipid bilayers: self-assembly and insertion. Journal of the Royal Society Interface. 2008;124:S241-S50.

[55] Grubmüller H, Heller H, Windemuth A, Schulten K. Generalized Verlet Algorithm for Efficient Molecular Dynamics Simulations with Long-Range Interactions. Molecular Simulation. 1991;6:121-42.

[56] Tuckerman M, Berne BJ, Martyna GJ. Reversible multiple time scale molecular dynamics. Journal of Chemical Physics. 1992;97:1990-2001. 
a

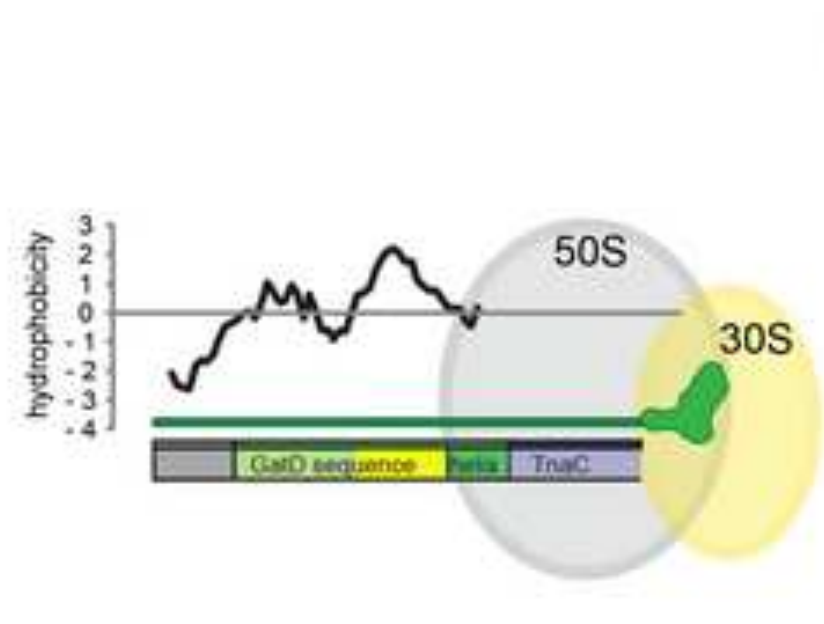

C

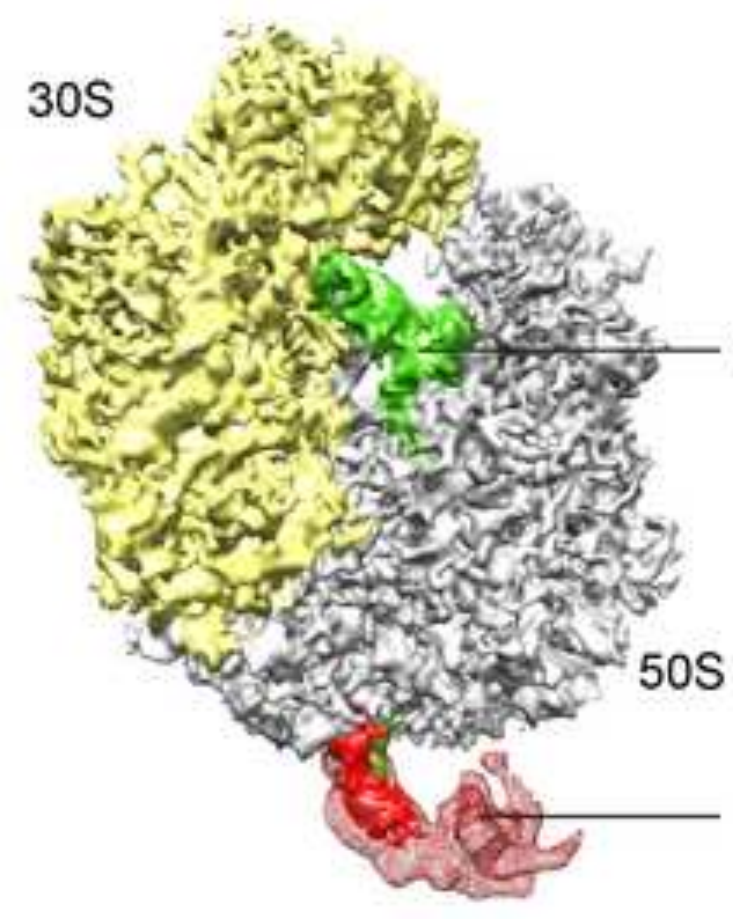

d

b
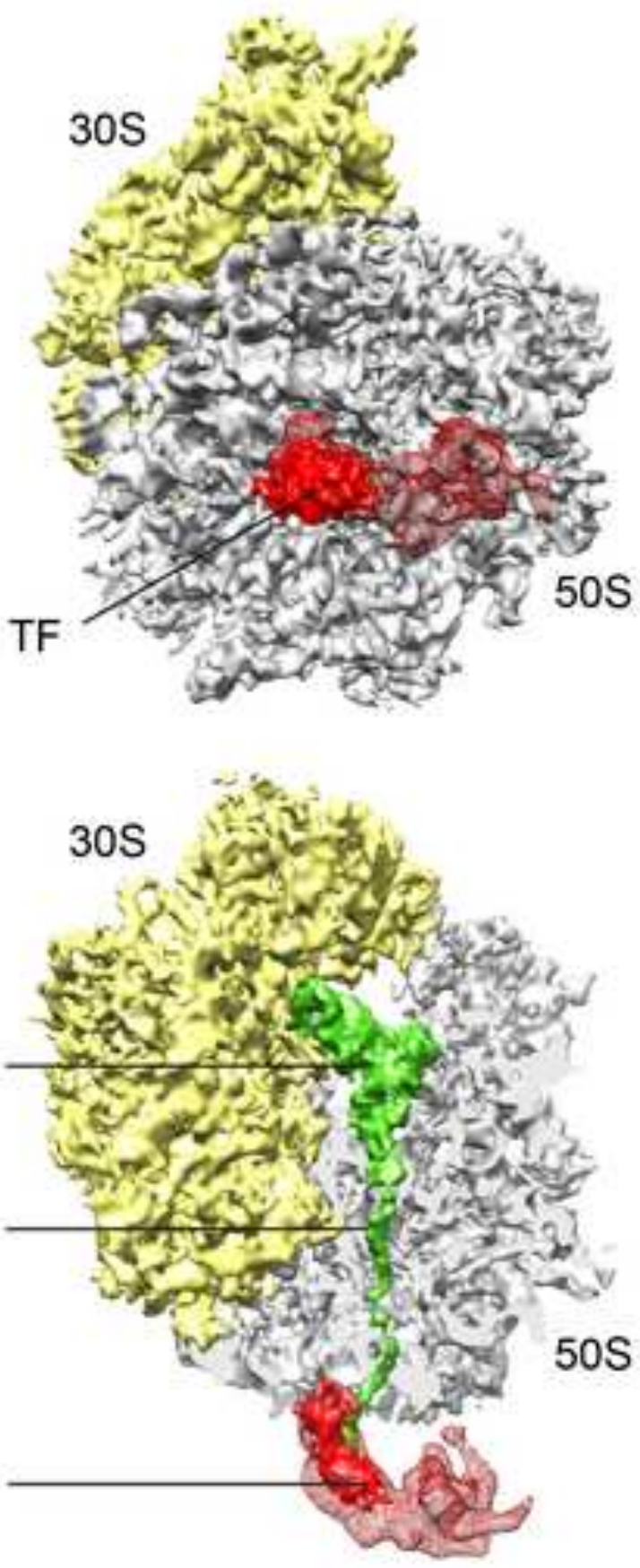
a

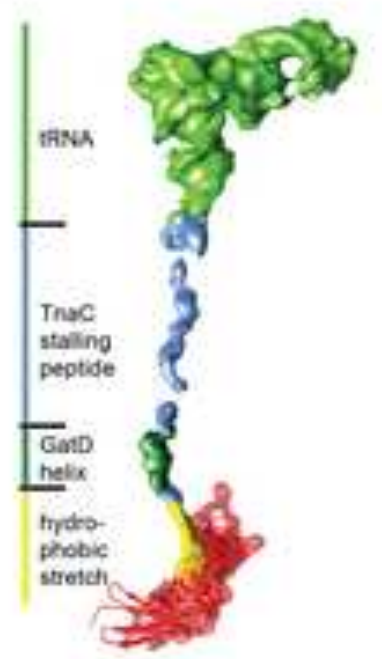

C

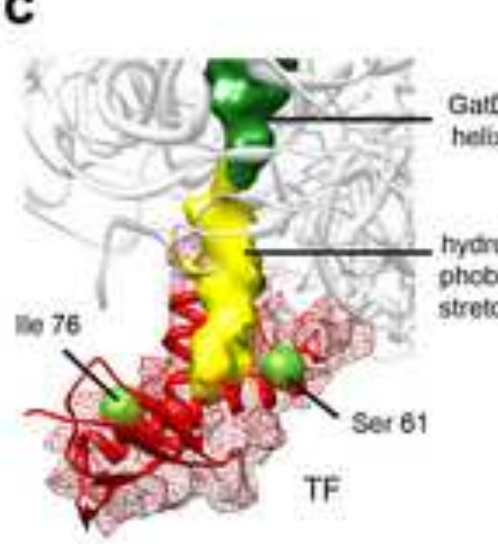

b

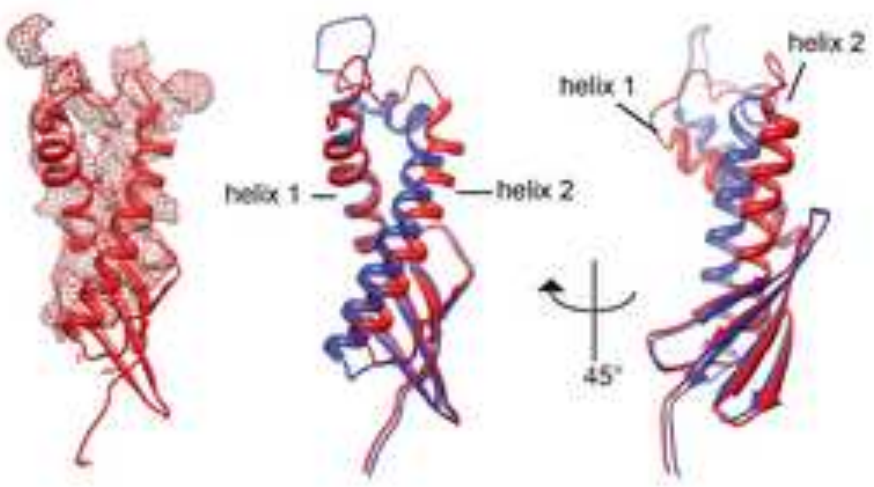

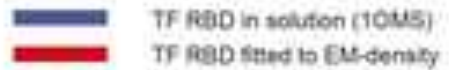

d
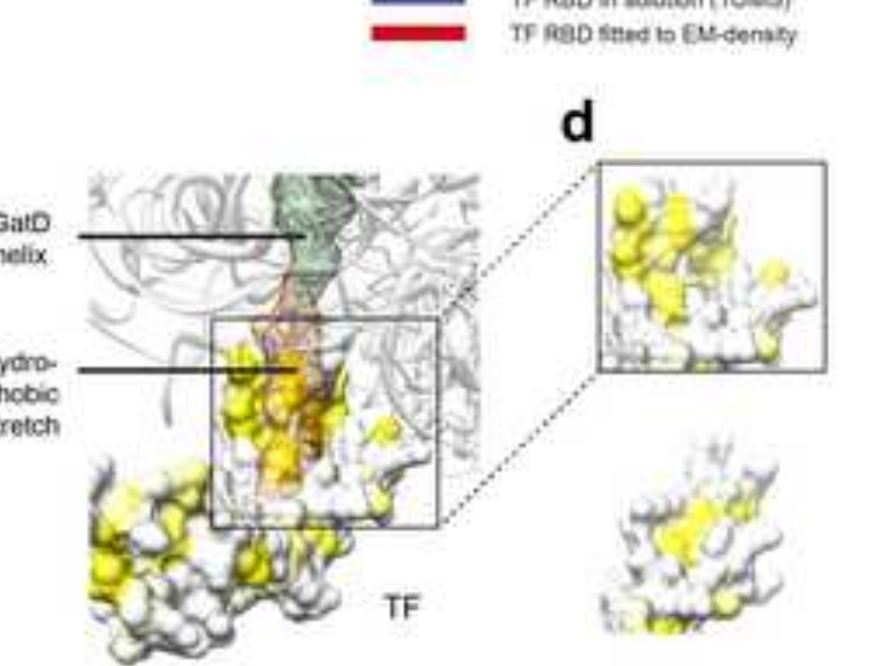

soy TF

Figure 2

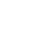


a
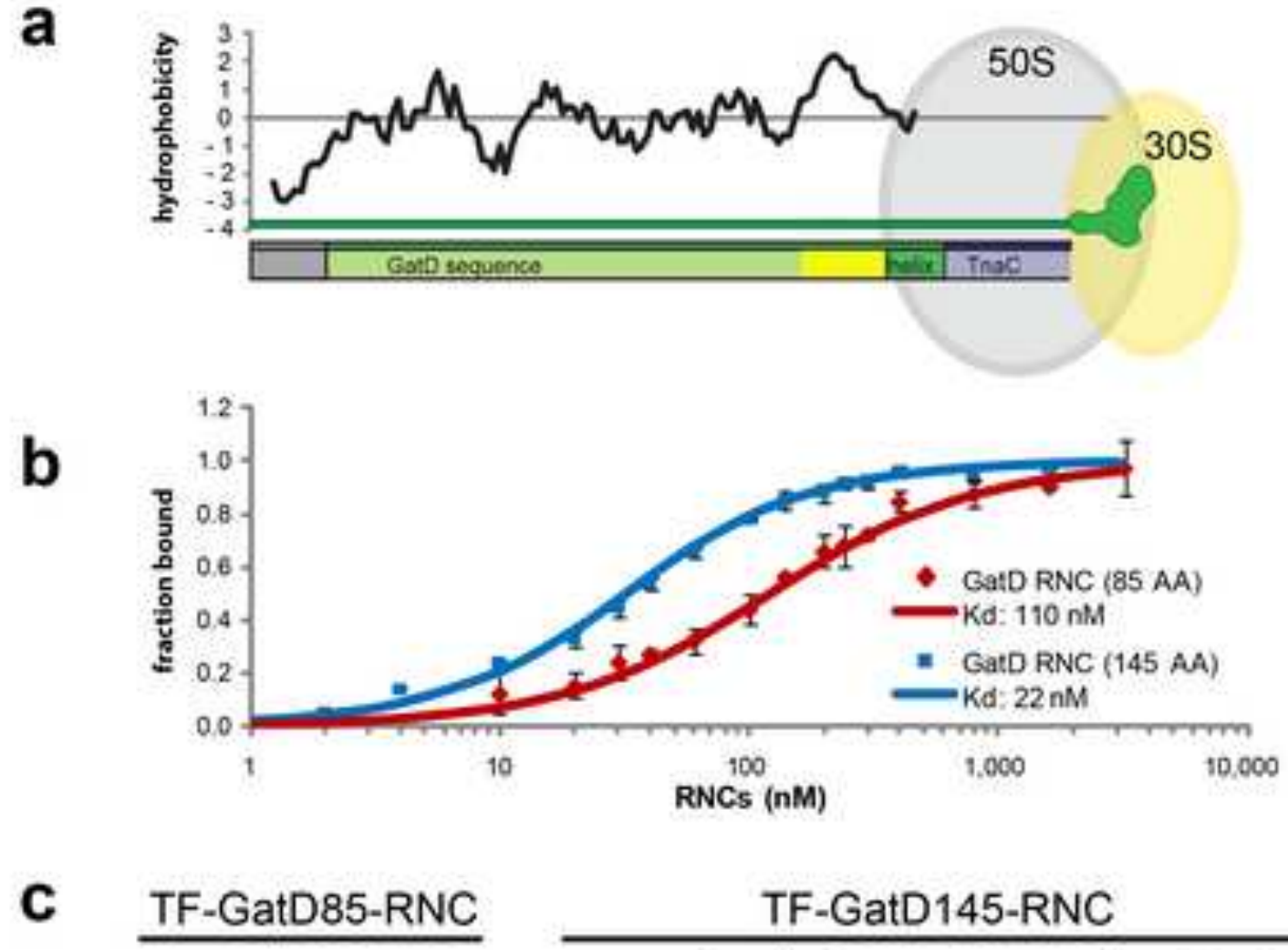

TF-GatD145-RNC

conformation 1

conformation 2
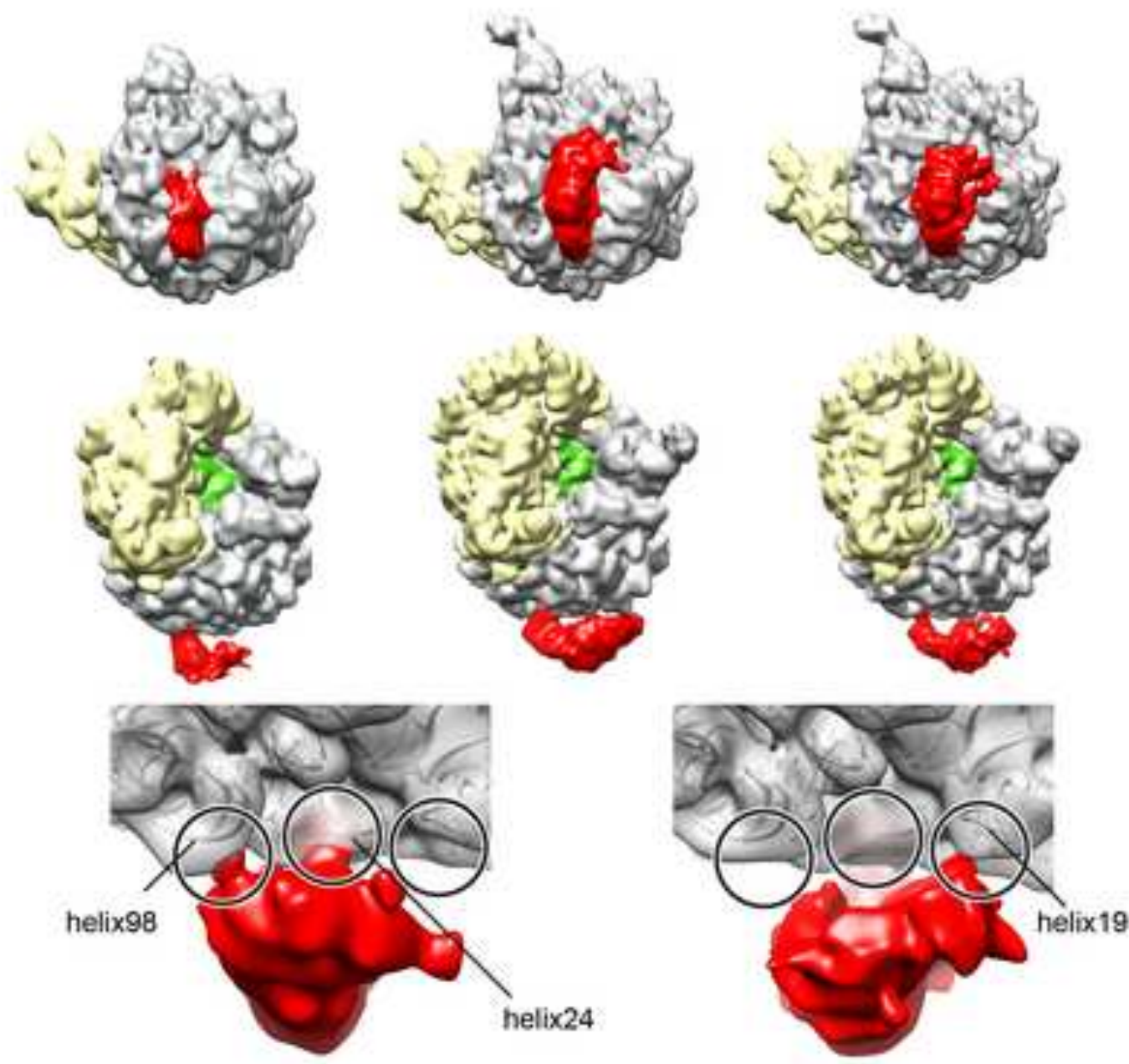
a

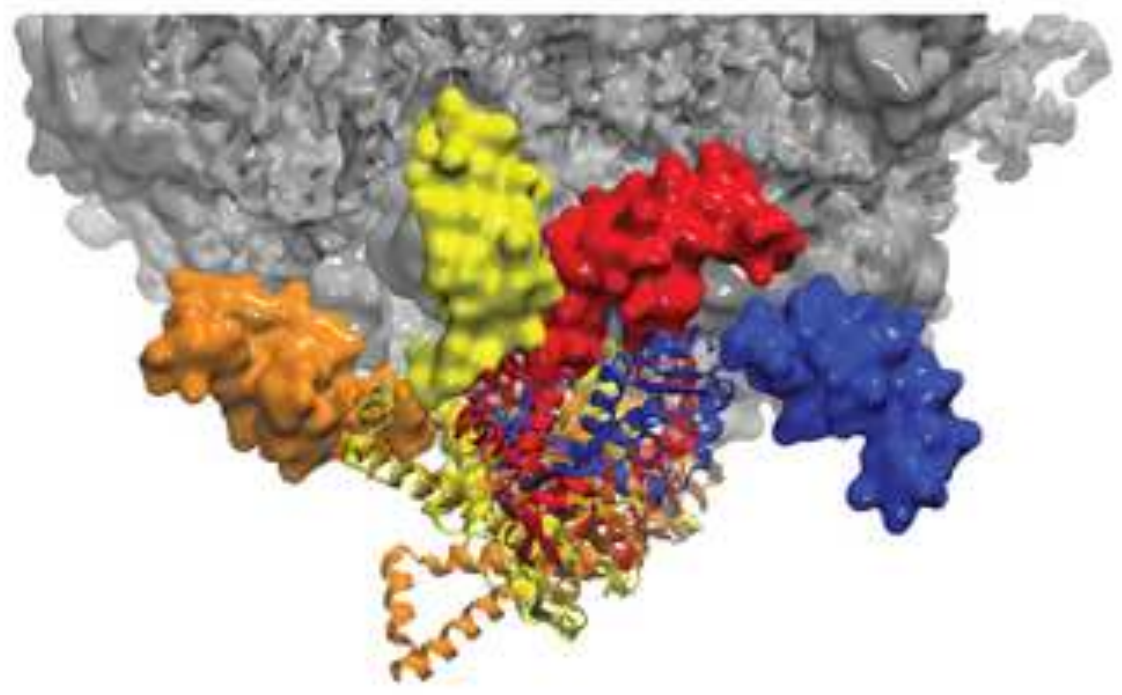

b TF-GatD145-RNC

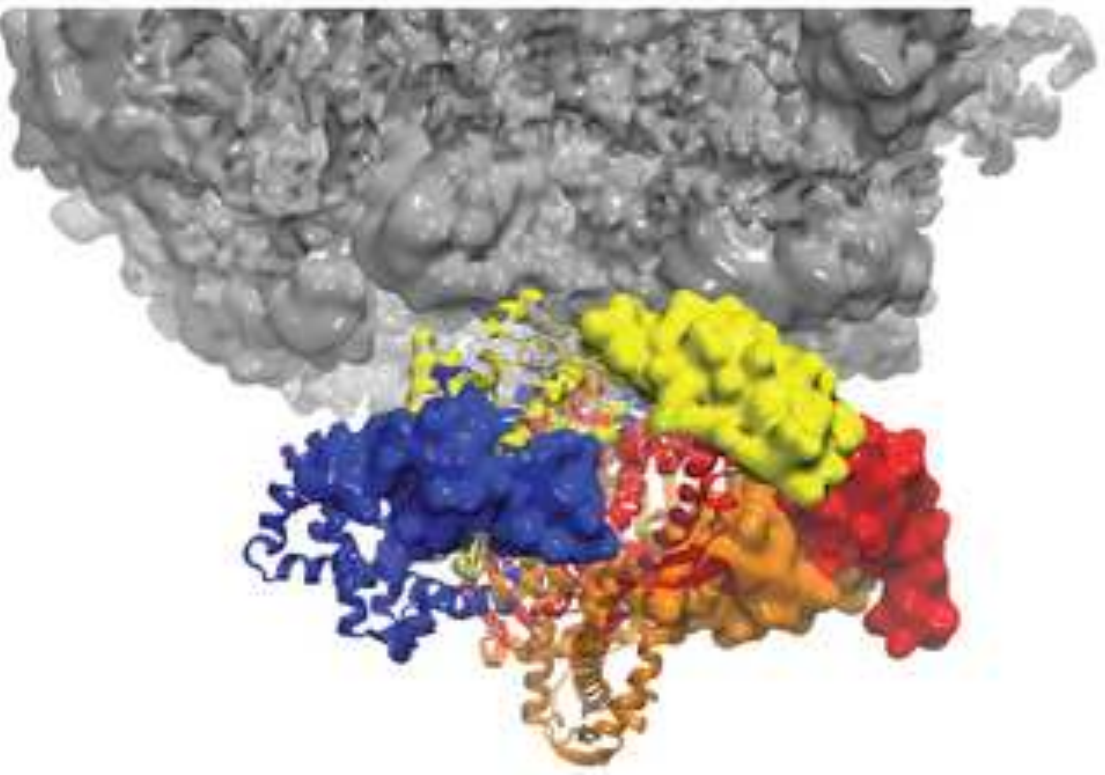

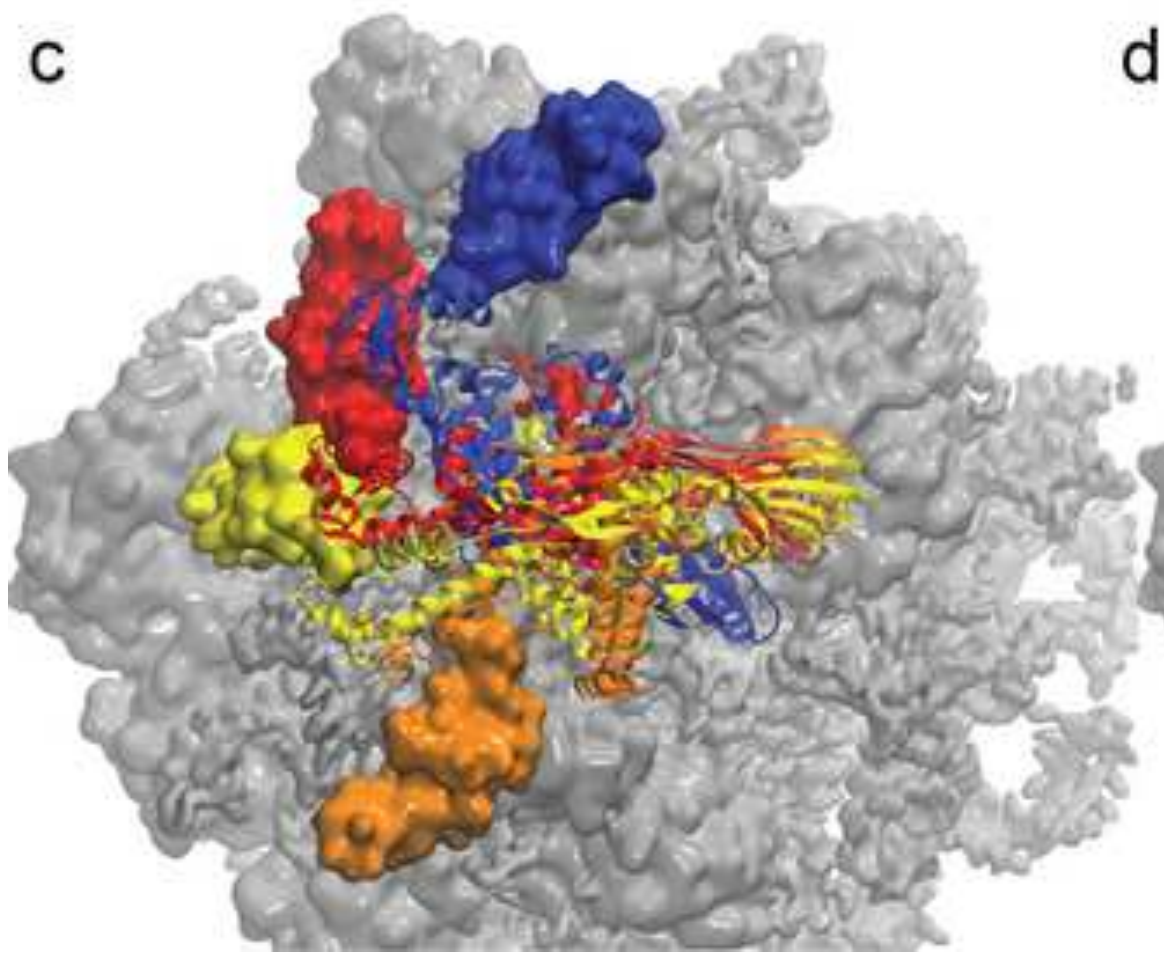

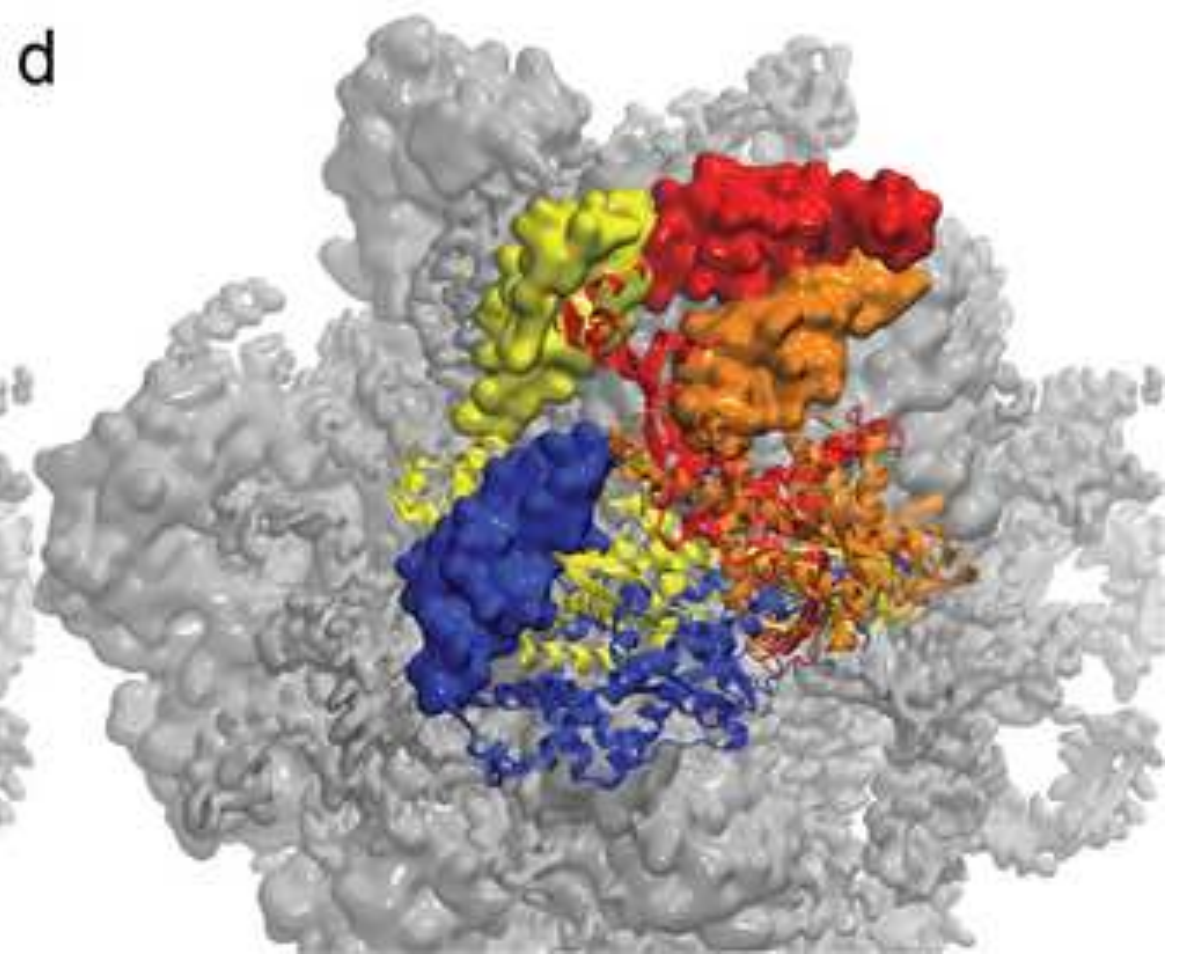



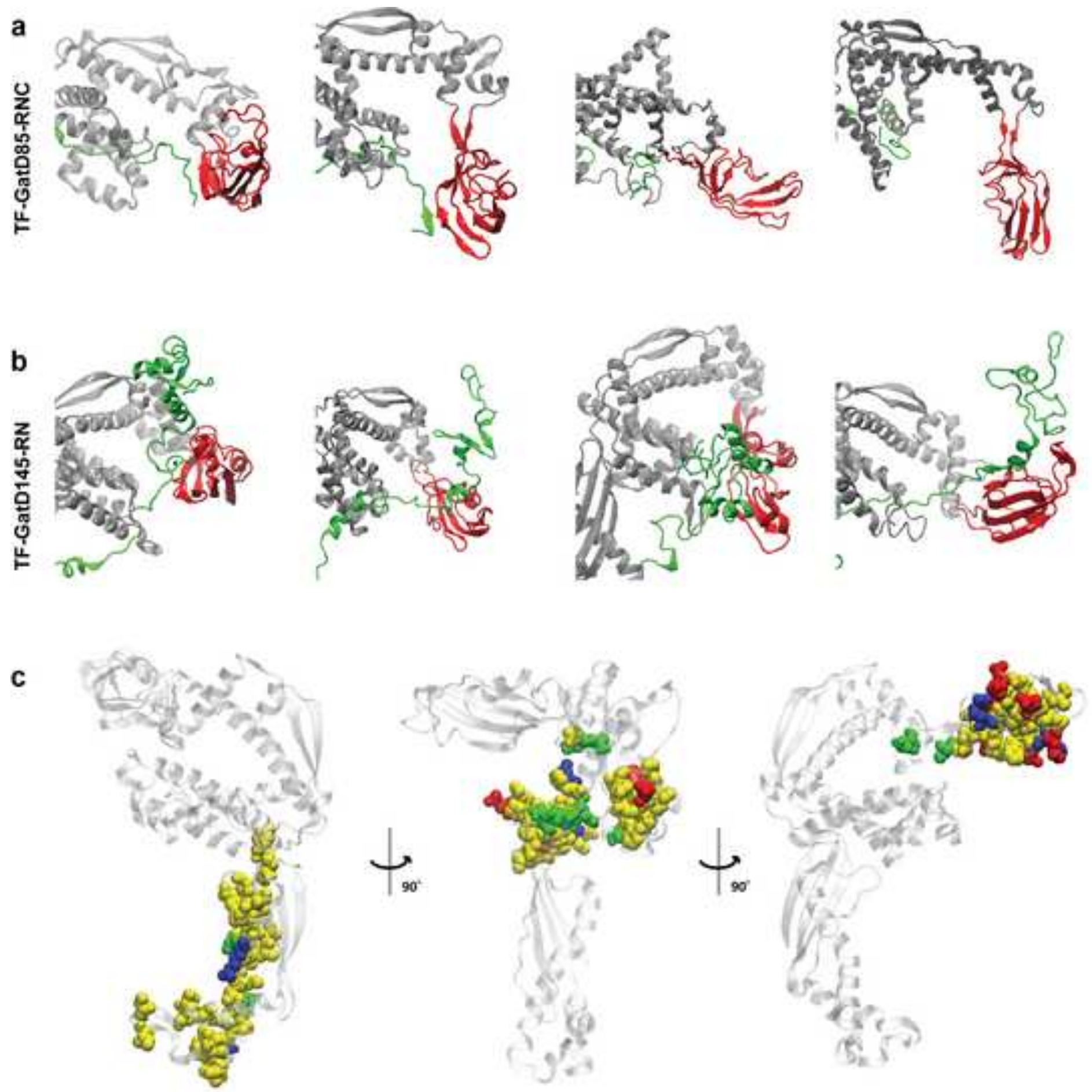

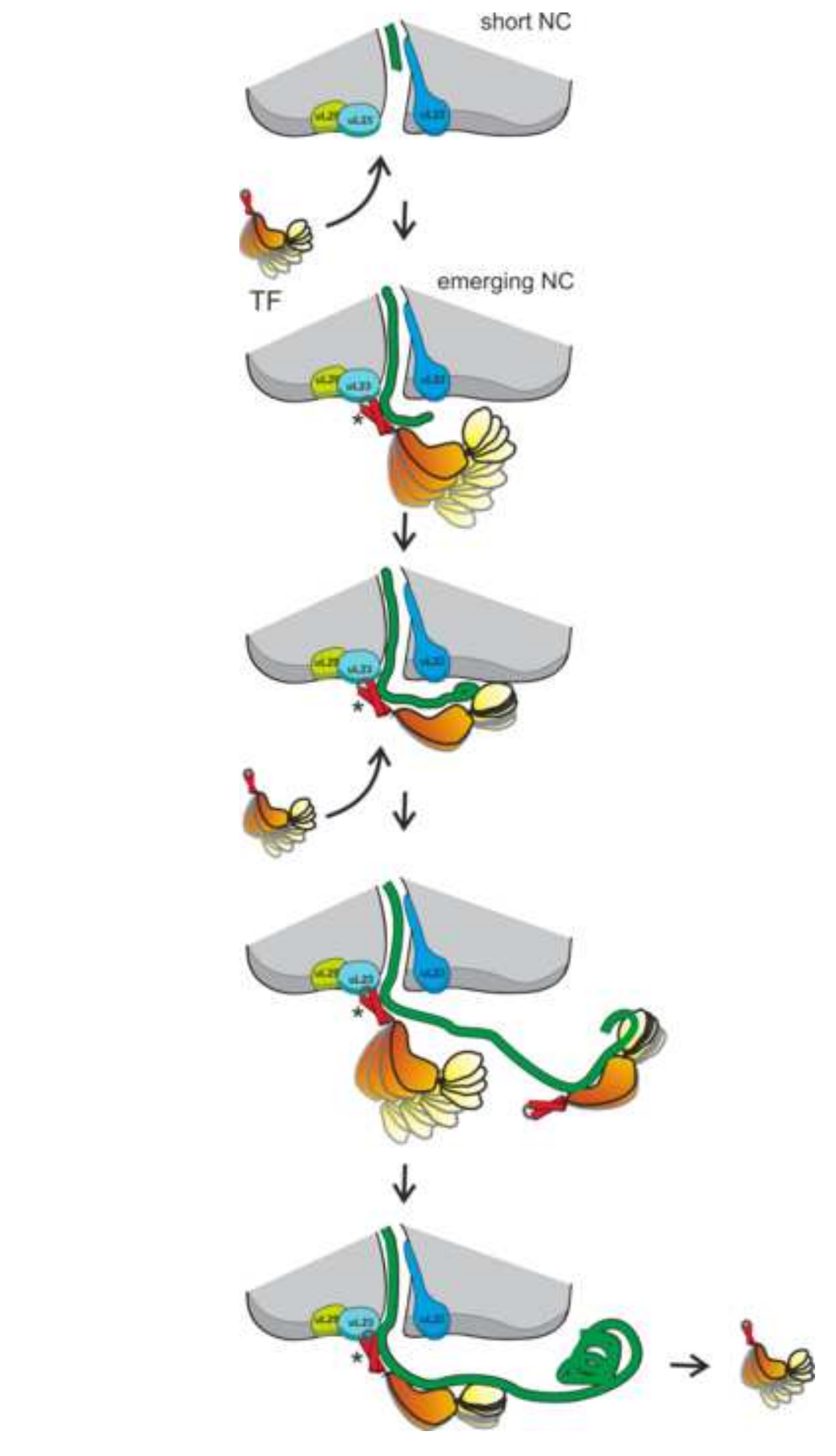

Figure 6

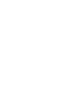



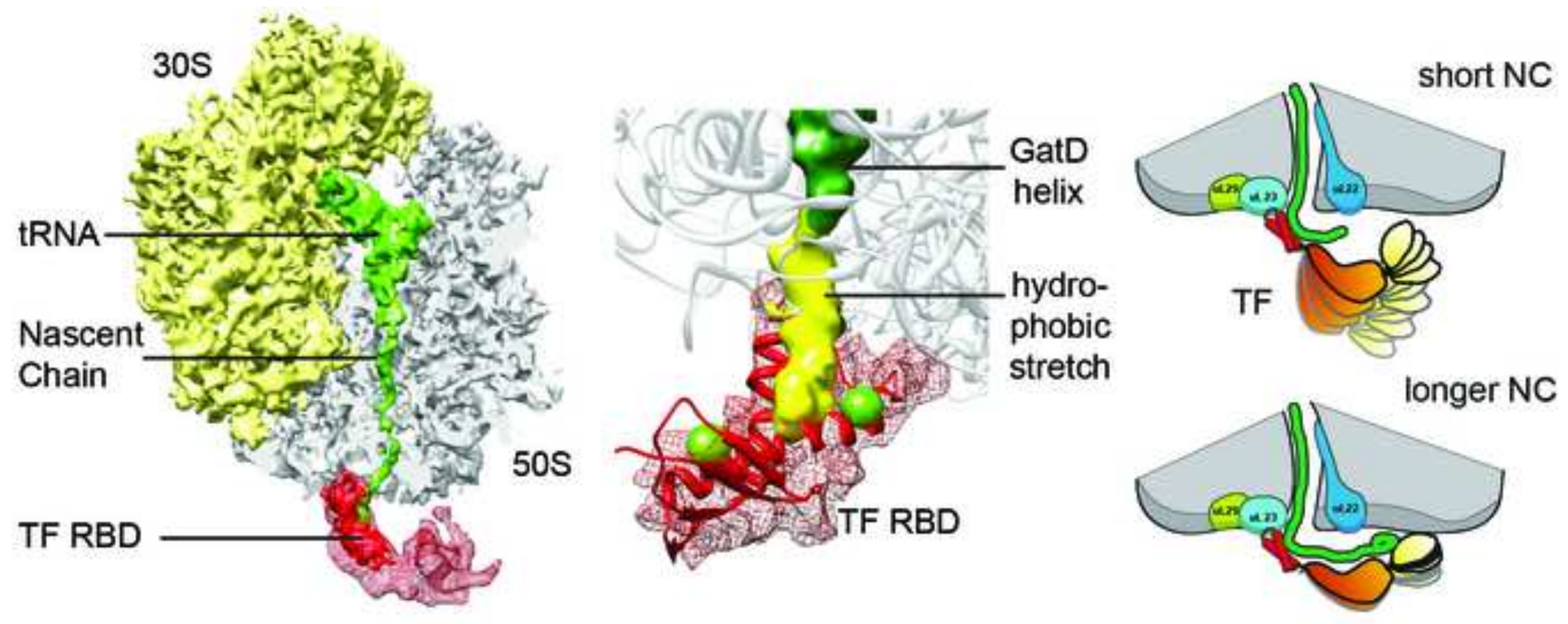